\title{
Thai traditional massage increases biochemical markers of bone formation in postmenopausal women: a randomized crossover trial
}

\author{
Sunee Saetung ${ }^{*}$, La-or Chailurkit and Boonsong Ongphiphadhanakul
}

\begin{abstract}
Background: The effect of massage therapy on bone metabolism in adults has only scarcely been explored. In a randomized crossover trial, we investigated the skeletal effect of Thai traditional massage by examining the changes in biochemical markers of bone turnover.

Methods: Forty-eight postmenopausal women participated in the study. All volunteers were randomized to a 2-hour session of Thai traditional massage twice a week for 4 weeks and a 4-week control period after a 2-week washout, or vice versa. Twenty-one subjects were allocated to receiving Thai traditional massage first, followed by the control period, while 27 were initially allocated to the control period.

Results: Serum P1NP increased significantly after Thai traditional massage $(P<0.01)$, while there was no change in serum osteocalcin or CTX. During the control period, there was no significant change in P1NP, osteocalcin or CTX compared to baseline. When age and height were taken into account, P1NP in postmenopausal women whose ages were in the middle and higher tertiles and whose heights were in the lower and middle tertiles $(n=22)$ had a $14.8 \pm 3.3 \%$ increase in P1NP after massage $(P<0.001)$, while no change in P1NP was found in the rest of the women $(n=26)$.

Conclusions: Thai traditional massage results in an increase in bone formation as assessed by serum P1NP, particularly in postmenopausal women who are older and have a smaller body build. Future studies with larger samples and additional design features are warranted.

Trial registration: ClinicalTrials.gov NCT01627028

Keywords: Bone formation marker, Postmenopausal women, Thai traditional massage
\end{abstract}

\section{Background}

Mechanical loading favorably influences bone mass. Active exercise, as well as passive exercise through lowamplitude whole-body vibration, have been demonstrated to improve bone mass or delay bone loss $[1,2]$. Massage therapy has been shown to alleviate bone pain [3] and improve bone growth in both animals [4] and humans [5] during the postnatal period. However, the effect of massage therapy on bone metabolism in adults has only scarcely been explored.

Thai Traditional massage exerts pressure on the body in a rhythmic fashion. The massage performer uses the

\footnotetext{
* Correspondence: ssaetung@hotmail.com

Department of Medicine, Ramathibodi Hospital, Rama 6 Rd, Rajthevi, Bangkok 10400, Thailand
}

outstretched heels of both hands to exert pressure on the body of the subject approximately once every 1-2 seconds for 2 hours. It is likely that the physical load from Thai traditional massage may induce strain in the skeleton and affect bone, similar to other means of applying mechanical load. We have demonstrated in a previous study that Thai traditional massage results in an acute anabolic effect on bone, as assessed by biochemical markers of bone turnover [6]. It is unclear if a longer term of massage therapy would result in similar effects. Toward this end, a randomized crossover study was employed to investigate the skeletal effect of Thai traditional massage by examining the changes in biochemical markers of bone turnover.

\section{Biomed Central}


Table 1 Full body Thai traditional massage protocol*

Subject position

1. Lying on back

2. Lying on side

3. Lying on chest
Massage area

Duration (minutes)

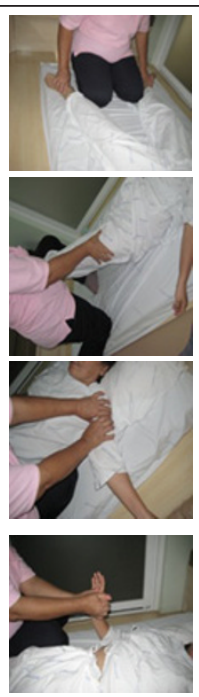

Feet- ankles

Leg line and stretch

Abdomen, shoulders and arms

Hands

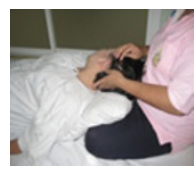

Neck, head and face

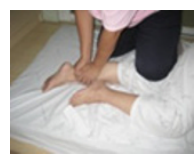

45

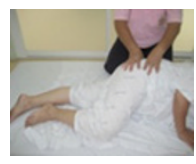

Hip and buttock

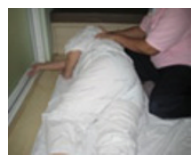

Spine, waist and back

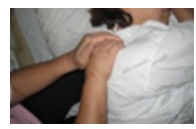

Shoulders, arms and hands

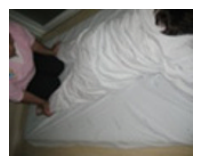

30

Feet- ankle

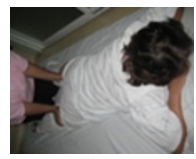

Leg line and stretch

Feet and legs

(1)

ras 


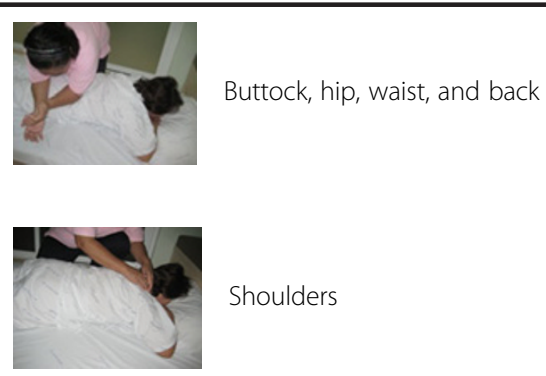

* Consents were obtained from both subjects in the pictures.

\section{Methods}

Subjects

A randomized crossover design was used. A total of 48 postmenopausal women participated in the study. All were non-diabetic, as defined by a 2-hour plasma glucose level $<200 \mathrm{mg} / \mathrm{dL}$ on a 75 -g oral glucose tolerance test. Subjects having disorders that could affect bone metabolism - such as hyperparathyroidism, thyrotoxicosis, diabetes, rheumatoid arthritis and cancer, as well as those who were taking glucocorticoids or medications for osteoporosis - were excluded (Additional file 1). The study was approved by the Institutional Review Board of Ramathibodi Hospital. Signed informed consent was obtained from each subject prior to the study. All subjects were enrolled and the study performed at the Endocrine and Metabolism Unit of Ramathibodi Hospital, Bangkok from March 2011 to March 2012.

\section{Thai traditional massage}

All volunteers were randomly assigned to either the treatment or the control group using a computer generated sequence. Subjects in the treatment group underwent a 2-hour session of Thai traditional massage twice a week for 4 weeks, while no intervention was given to subjects in the control group. After a 2-week washout period, subjects were switched to the other arm of intervention for 4 weeks.

Thai traditional massage was performed by a single masseuse throughout the study. Subjects were requested to change into comfortable, loose-fitting clothes and to lie flat on a firm mattress on the floor. The procedure consisted of the masseuse applying firm, rhythmic pressure over the volunteer's body through the heels of her hands. The 2-hour procedure started with massaging the feet, and then the legs, arms, hands, back and neck, ending with a head massage (Table 1).

\section{Biochemical measurement}

Subjects were requested to refrain from exercise for 24 hours and to fast for at least 10 hours before blood was drawn in the morning. Blood samples were collected on the day of the initiation of the massage or control period, and on the day following each massage or control period. All samples were stored at -80 Celsius and analyzed in batch at the end of the study. Serum Cterminal telopeptide of type I collagen $(\mathrm{CTx}-\mathrm{I})$, total procollagen type 1 amino-terminal propeptide (P1NP), N-MID osteocalcin, and insulin were determined by electrochemiluminescence immunoassay on a Cobas e 411 analyzer (Roche Diagnostics, Mannheim, Germany). The intra-assay precision was $3.8 \%, 3.8 \%, 1.4 \%$ and $1.9 \%$, respectively.

\section{Bone densitometry and measurement of body composition}

Bone mineral density (BMD) and body composition were measured by dual-energy X-ray absorptiometry (DEXA) (Lunar Prodigy; GE Healthcare, Little Chalfont, UK). Daily calibration and quality control were performed regularly according to the manufacturer's recommendations. Body composition and BMD of lumbar spine 1-4, femoral neck and total hip were measured in each subject.

\section{Statistical analysis}

Changes in biochemical markers of bone turnover for both Thai traditional massage and control periods were assessed by paired Student's $t$-test. Crossover statistical analysis was performed by the pkcross routine in Stata 12 software (StataCorp LP, College Station, TX), assuming no carryover effects. A $P$-value of less than 0.05 was considered statistically significant.

\section{Results}

Table 2 demonstrates the clinical characteristics of the 48 study subjects. The mean age was $59.1 \pm 4.3$ years. All were postmenopausal women with an average of $8.9 \pm 5.6$ years since menopause. Seventeen (35.4\%) were obese, based on BMI $>25 \mathrm{~kg} / \mathrm{m}^{2}$. Seven (14.6\%) had osteoporosis either at the spine, femoral neck or total hip, according to a DEXA T-score of -2.5 or less at the corresponding sites.

Twenty-one subjects were allocated to have Thai traditional massage first, followed by the control period, while 
Table 2 Characteristics of the study population

\begin{tabular}{ll}
\hline Parameters & Mean \pm SD (range) $(\mathbf{n}=\mathbf{4 8})$ \\
\hline Age (years) & $59.1 \pm 4.4(49.8-66.6)$ \\
Years since menopause & $8.9 \pm 5.6(1.2-24.3)$ \\
Body weight $(\mathrm{kg})$ & $57.1 \pm 7.4(40.0-73.8)$ \\
Height $(\mathrm{cm})$ & $153.1 \pm 5.0(141.5-163.5)$ \\
Body mass index $\left(\mathrm{kg} / \mathrm{m}^{2}\right)$ & $24.3 \pm 2.9(18.1-30.9)$ \\
\hline
\end{tabular}

27 were initially allocated to the control period. When combined data from all subjects were analyzed, it was found that serum P1NP increased significantly after Thai traditional massage while there was no change in serum osteocalcin or CTX. During the control period, there was no significant change in P1NP, osteocalcin or CTX compared to baseline (Table 3). In a linear mixed model looking at the effect of massage and the sequence of intervention, it was found that Thai traditional massage significantly increased serum P1NP. No influence of the sequence of treatment allocation was found (Table 4).

It is likely that body size as well as bone and fat mass may affect the responsiveness to the externally applied mechanical loading from Thai traditional massage. We investigated if there were associations between the percent change in P1NP after massage and age, body weight, height, and body composition. Table 5 demonstrates the change in serum P1NP according to body height tertiles. There was a significant increase in serum P1NP post-massage in subjects in both the lower and middle height tertiles. However, no change in serum P1NP was detected in subjects in the upper body height tertile. When age and body height were considered in combination, it was found that P1NP in postmenopausal women whose ages were in the middle and upper tertiles and whose heights were in the lower and middle tertiles $(n=22)$ had a $14.8 \pm 3.3 \%$ increase in P1NP after massage $(P<0.001)$, while no change in P1NP was found in the rest of the women $(\mathrm{n}=26)$.

Table 3 Serum P1NP (median (range)), osteocalcin (mean \pm SE) and CTX (median (range)) before and after massage or the control period

\begin{tabular}{lccc}
\hline & Baseline & After massage & $\boldsymbol{P}$ \\
\hline P1NP & $45.2(40.2-50.3)$ & $48.5(44.2-53.3)$ & $<0.01$ \\
Osteocalcin & $22.8 \pm 1.0$ & $22.9 \pm 0.8$ & NS \\
CTX & $0.38(0.33-0.43)$ & $0.39(0.34-0.43)$ & NS \\
& Baseline & After control period & $\boldsymbol{P}$ \\
P1NP & $45.8(41.9-50.1)$ & $46.0(42.0-50.4)$ & NS \\
Osteocalcin & $23.0 \pm 0.9$ & $23.0 \pm 0.9$ & NS \\
CTX & $0.38(0.34-0.43)$ & $0.38(0.34-0.43)$ & NS \\
\hline
\end{tabular}

Serum P1NP increased significantly after massage.
Table 4 Effects of treatment and its sequence on the change in P1NP after massage

\begin{tabular}{lccc}
\hline & MS & F & $\boldsymbol{P}$ \\
\hline Sequence effect & 43.76 & 0.26 & NS \\
Treatment effect & 1294.95 & 5.69 & $<0.05$ \\
\hline
\end{tabular}

The effect of massage was statistically significant, while the sequence of treatment did not have a significant effect.

\section{Discussion}

Massage has been widely utilized for the alleviation of a number of musculoskeletal disorders, including low back pain and bone pain from metastatic malignancy [3]. Despite its common utilization, studies showing evidence of the beneficial effects of massage therapy are still limited. Thai traditional massage has been shown to reduce pain and muscle tension in patients with scapulocostal syndrome [7]. Moreover, in a preliminary study on the acute effects of Thai traditional massage on biochemical markers of bone turnover, we demonstrated that a single 2-hour session can acutely increase serum P1NP, a marker of bone formation by $4.8 \%$ [6]. Using a randomized crossover design we demonstrated in the present study that two sessions per week of Thai traditional massage for 4 weeks resulted in a higher increase in P1NP, particularly in older individuals with smaller body built. The magnitude of change in P1NP from Thai traditional massage was comparable to the change in serum alkaline phosphatase in a study using vibration platform [8] but was much less than that achieved with parathyroid hormone, a potent bone formation agent used in the treatment of osteoporosis, which usually reaches $100 \%$ or higher [9]. Osteocalcin did not change significantly in the present study despite the changes in P1NP. Studies with bone forming agents such as parathyroid hormone and sclerostin monoclonal antibody have shown that osteocalcin is a less sensitive marker in response to treatments [10,11]. It is of note that the effect of Thai traditional massage on bone formation was more apparent in postmenopausal women

Table 5 Changes in P1NP (\%) after massage according to body height and age tertiles

\begin{tabular}{ccc}
\hline Body height & Post-massage change in P1NP & $\boldsymbol{P}$ \\
\hline Tertile 1 & $+12.3 \pm 3.9$ & $<0.01$ \\
Tertile 2 & $+9.7 \pm 4.0$ & $<0.05$ \\
Tertile 3 & $+3.5 \pm 3.5$ & NS \\
Age & Post-massage change in P1 NP & $\boldsymbol{P}$ \\
Tertile 1 & $+3.9 \pm 3.2$ & NS \\
Tertile 2 & $+13.6 \pm 3.8$ & $<0.01$ \\
Tertile 3 & $+8.1 \pm 4.3$ & 0.08 \\
\hline
\end{tabular}

Serum P1NP increased significantly in the middle and lower body height tertiles. Likewise, serum P1NP increased significantly in the middle age tertile. The increase in P1NP in the upper age tertile almost reached statistical significance. 
of older age. While the improvement in the marker of bone formation cannot readily be extrapolated to an enhancement of bone mass or reduced fracture risk, our finding at least suggests that Thai traditional massage is likely to be beneficial to bone, particularly in women of advancing age among whom osteoporosis is a common health problem.

The effect of massage on bone metabolism in adults has scarcely been explored. However, a number of studies have investigated the influence of massage on the alteration of bone growth, particularly during the postnatal period. For example, massage in the early postnatal period was found to promote lean mass and bone growth in experimental animals [4]. In humans, when combined with physical activity, massage during the peri-neonatal period improves bone formation without changes in bone resorption [12]. Our findings are in keeping with those in infants, where massage therapy results in an increase in serum P1NP but not CTX.

It is well established that mechanical load affects bone cells. The strain characteristics that determine skeletal responses include strain magnitude [13], strain frequency [14] and strain rate [15]. There appears to be an inverse relationship between strain magnitude and frequency for inducing osteogenic effects. Low-magnitude mechanical load needs to be applied at high frequency in order to have an effect equivalent to high-magnitude mechanical load at lower frequency [16]. Moreover, the duration and cycle number of the loading are other factors related to the increase in osteoblast proliferation $[17,18]$. We showed in the present study that body height was inversely related to the increased in P1NP after Thai traditional massage. It is likely that this is partly because of tall postmenopausal women received less repetitive cyclic numbers of massage per body surface area than shorter women during the same period. In line with our previous study, we demonstrated in the present study that external periodic mechanical loading applied through Thai traditional massage is likely to have an anabolic effect on bone; this suggests that Thai traditional massage could be another option for enhancing bone health through mechanical loading.

Besides its direct effect on bone through mechanical loading, it is conceivable that Thai traditional massage may affect bone indirectly through the central nervous system. The adipokine leptin inhibits bone formation through a central nervous system delay [19]. Mechanical tactile stimulation reduces stress hormones and improves bone mineralization in rats [20]. Moreover, a study using functional MRI has shown that types of massage can influence brain cortical areas differently [21]. To what extent the effect of Thai traditional massage is due to its effect on stress hormones and the central nervous system is unknown. One of the other possible mechanisms involves ghrelin. It has been shown that ghrelin enhances bone formation [22], and that massage therapy in infants increases circulating ghrelin [23].

There are a number of limitations in the present study. The sample size is relatively small and may not be able to detect small effects on bone resorption. Moreover, although the current results are consistent with those of our previous study and of other studies in infants, it is still unclear if the increase in bone formation as reflected by bone markers will result in higher bone mass or reduced fractures. It is of note that only a single masseur performed the Thai traditional massage throughout the study. The result may not be readily generalized to other masseurs. Further studies, which include multiple practitioners, to confirm our results, as well as to investigate the effect of Thai traditional massage on bone mass or fractures, are warranted.

\section{Conclusions}

The present study demonstrated that Thai traditional massage results in an increase in bone formation as assessed by serum P1NP, particularly in postmenopausal women who are older and have a smaller body build. Future studies with larger samples and additional design features are warranted.

\section{Additional file}

Additional file 1: CONSORT 2010 Flow Diagram.

\section{Competing interests}

All authors have no competing interests.

\section{Authors' contributions}

$\mathrm{BO}$ conceived the idea of this study and drafted the manuscript. SS participated in its design and coordination and helped to draft the manuscript. LC performed the electrochemiluminescence immunoassay. All authors read and approved the final manuscript.

\section{Acknowledgements}

This research was supported by the Thailand Research Fund. We would like to thank all the volunteers and the masseur who performed the massages throughout the study.

Received: 26 July 2012 Accepted: 24 January 2013

Published: 25 March 2013

\section{References}

1. Chow RHJE, Notarius C: Effect of two randomised exercise programmes on bone mass of healthy postmenopausal women. Br Med J (Clin Res Ed) 1887, 295:1441-1444.

2. Rubin C, Recker R, Cullen D, Ryaby J, McCabe J, McLeod K: Prevention of postmenopausal bone loss by a low-magnitude, high-frequency mechanical stimuli: a clinical trial assessing compliance, efficacy, and safety. J Bone Miner Res 2004, 19(3):343-351.

3. Jane SW, Chen SL, Wilkie DJ, Lin YC, Foreman SW, Beaton RD, Fan JY, Lu MY, Wang YY, Lin YH, et al: Effects of massage on pain, mood status, relaxation, and sleep in Taiwanese patients with metastatic bone pain: a randomized clinical trial. Pain 2011, 152(10):2432-2442.

4. Chen H, Miller S, Shaw J, Moyer-Mileur L: Massage therapy during early postnatal life promotes greater lean mass and bone growth, mineralization, and strength in juvenile and young adult rats. J Musculoskelet Neuronal Interact 2009, 9(4):278-287. 
5. Aly H, Moustafa MF, Hassanein SM, Massaro AN, Amer HA, Patel K: Physical activity combined with massage improves bone mineralization in premature infants: a randomized trial. J Perinatol 2004, 24(5):305-309.

6. Saetung S, Chailurkit LO, Ongphiphadhanakul B: Acute changes in biochemical markers of bone resorption and formation after Thai traditional massage. J Med Assoc Thai 2010, 93(7):771-775.

7. Buttagat V, Eungpinichpong W, Chatchawan U, Arayawichanon P: Therapeutic effects of traditional Thai massage on pain, muscle tension and anxiety in patients with scapulocostal syndrome: a randomized single-blinded pilot study. J Bodyw Mov Ther 2012, 16(1):57-63.

8. Bemben DA, Palmer IJ, Bemben MG, Knehans AW: Effects of combined whole-body vibration and resistance training on muscular strength and bone metabolism in postmenopausal women. Bone 2010, 47(3):650-656.

9. Quesada-Gomez JM, Muschitz C, Gomez-Reino J, Greisen H, Andersen HS, Dimai HP: The effect of PTH(1-84) or strontium ranelate on bone formation markers in postmenopausal women with primary osteoporosis: results of a randomized, open-label clinical trial. Osteoporos Int 2011, 22(9):2529-2537.

10. Sikjaer T, Rejnmark L, Rolighed L, Heickendorff L, Mosekilde L: The effect of adding PTH(1-84) to conventional treatment of hypoparathyroidism: a randomized, placebo-controlled study. J Bone Miner Res 2011, 26(10):2358-2370.

11. Padhi D, Jang G, Stouch B, Fang L, Posvar E: Single-dose, placebocontrolled, randomized study of AMG 785, a sclerostin monoclonal antibody. J Bone Miner Res 2011, 26(1):19-26.

12. Moyer-Mileur $\sqcup$, Ball SD, Brunstetter VL, Chan GM: Maternal-administered physical activity enhances bone mineral acquisition in premature very low birth weight infants. J Perinatol 2008, 28(6):432-437.

13. Rubin $C T$, Lanyon LE: Regulation of bone mass by mechanical strain magnitude. Calcif Tissue Int 1985, 37(4):411-417.

14. Qin YX, Rubin CT, McLeod KJ: Nonlinear dependence of loading intensity and cycle number in the maintenance of bone mass and morphology. J Orthop Res 1998, 16(4):482-489.

15. Turner $\mathrm{CH}$, Owan I, Takano Y: Mechanotransduction in bone: role of strain rate. Am J Physiol 1995, 269(3 Pt 1):E438-E442.

16. Cullen DM, Smith RT, Akhter MP: Bone-loading response varies with strain magnitude and cycle number. J Appl Physiol 2001, 91(5):1971-1976.

17. Nagatomi J, Arulanandam BP, Metzger DW, Meunier A, Bizios R: Frequencyand duration-dependent effects of cyclic pressure on select bone cell functions. Tissue Eng 2001, 7(6):717-728.

18. Kaspar D, Seidl W, Neidlinger-Wilke C, Beck A, Claes L, Ignatius A: Proliferation of human-derived osteoblast-like cells depends on the cycle number and frequency of uniaxial strain. J Biomech 2002, 35(7):873-880

19. Karsenty G, Oury F: The central regulation of bone mass, the first link between bone remodeling and energy metabolism. J Clin Endocrinol Metab 2010, 95(11):4795-4801.

20. Haley S, O'Grady S, Gulliver K, Bowman B, Baldassarre R, Miller S, Lane RH, Moyer-Mileur L: Mechanical-tactile stimulation (MTS) intervention in a neonatal stress model improves long-term outcomes on bone. J Musculoskelet Neuronal Interact 2011, 11(3):234-242.

21. Sliz D, Smith A, Wiebking C, Northoff G, Hayley S: Neural correlates of a single-session massage treatment. Brain Imaging Behav 2012, 6(1):77-87.

22. Fukushima N, Hanada R, Teranishi H, Fukue $Y$, Tachibana T, Ishikawa $H$, Takeda S, Takeuchi Y, Fukumoto S, Kangawa K, et al: Ghrelin directly regulates bone formation. J Bone Miner Res 2005, 20(5):790-798.

23. Kim SHKE, Park SK: Effect of Massage Therapy on Growth of Preterm Infants: Relationship with Ghrelin, Leptin and Bone Specific Alkaline Phosphatase Levels. J Korean Soc Pediatr Endocrinol 2005, 10(2):169-175.

doi:10.1186/1472-6882-13-69

Cite this article as: Saetung et al:: Thai traditional massage increases biochemical markers of bone formation in postmenopausal women: a randomized crossover trial. BMC Complementary and Alternative Medicine 2013 13:69.

\section{Submit your next manuscript to BioMed Central and take full advantage of:}

- Convenient online submission

- Thorough peer review

- No space constraints or color figure charges

- Immediate publication on acceptance

- Inclusion in PubMed, CAS, Scopus and Google Scholar

- Research which is freely available for redistribution

Submit your manuscript at www.biomedcentral.com/submit
Ciomed Central 